Results 10,813 patients were identified (55\% male, mean (SD) age 71.07 ( \pm 10.48$), \mathrm{FEV}_{1} \%$ predicted $59.96 \%$ ( $\left.\pm 19.98 \%\right)$.

1677 deaths (15.5\%) occurred during the follow-up period. Compared with individuals with a normal BMI, underweight subjects had a higher mortality risk in adjusted analysis $(\mathrm{HR}=$ $1.58,95 \% \mathrm{CI}=1.31-1.88)$. The lowest mortality rates were in overweight subjects $(\mathrm{HR}=0.72,95 \% \mathrm{CI}=0.64-0.81)$ and very obese subjects had no significant difference $(\mathrm{HR}=0.83$, 95\% CI $=0.68-1.02, \mathrm{p}=0.08)$.

The relationship between hospitalisation rate and BMI was ' $U$ ' shaped. Admission rates were highest in the underweight category where $13.3 \%$ of subjects had $\geq 2$ admissions compared to $6.2 \%$ and $5.3 \%$ of overweight and obese subjects respectively.

A similar relationship was observed between BMI and respiratory-cause emergency department attendance. 13.9\% of underweight subjects had $\geq 2$ emergency department attendances. The lowest attendance rates were observed in overweight and obese subjects where $6.5 \%$ and $5.6 \%$ of subjects had $\geq 2$ attendances. Conclusions Underweight COPD patients have the highest death and hospitalisation rates, whilst being overweight or obese appears to have protective effects. There is potential for nutritional supplementation interventions in underweight COPD patients to improve outcomes, and further research into the protective effects of obesity is required.

\section{S123 CORONARY ATHEROSCLEROSIS DETECTED AT ELECTIVE ANGIOGRAPHY IS MORE SEVERE IN PEOPLE WITH COPD THAN IN THOSE WITHOUT}

SV Ruickbie, A Prasad, PW Jones, EH Baker. St George's University of London, London, UK 10.1136/thoraxjnl-2015-207770.129

Introduction Coronary artery disease (CAD) affects 16-53\% people with chronic obstructive pulmonary disease (COPD) and is the cause of death in $\sim 25 \%$ (Smith and Wrobel. Int J COPD. 2014;9:871-888). People with COPD have both high prevalence of cardiovascular risk factors and increased systemic inflammation and oxidative stress that can drive atherosclerosis. We therefore tested the hypothesis that patients with COPD have more extensive coronary artery disease compared to those without.

Methods All patients attending for elective coronary angiography March-July 2015 were invited to take part in a cross-sectional, observational study. Participants who gave consent underwent clinical assessment and spirometry prior to the procedure. COPD was defined as $\mathrm{FEV}_{1} / \mathrm{FVC} 10$ pack-year smoking history. CAD burden was quantified from the angiogram using the Gensini score (Neeland et al. Am Heart J 2012;164:547-552). A single rater (Professor of Interventional Cardiology), blinded to clinical diagnosis, determined number and severity of lesions. Blinded repeats were performed and ratings compared to clinical reports to ensure reliability. A nonlinear score was assigned to each lesion based on severity of stenosis and a multiplier applied depending on lesion location in the coronary tree. Lesion scores were summed to derive Gensini score which was log-transformed for analysis.

Results 249 of 294 (85\%) people approached took part, 46 (19\%) had COPD. The Table 1 compares demographic, respiratory disease-related and cardiovascular risk factors between people with and without COPD. Gensini score was higher in COPD patients $(22.5(8.5-46.0))$ than in those without (12.5 (6.0$26.8), \mathrm{p}=0.04)$, indicating greater burden of coronary atheroma. COPD patients had more circumflex lesions and tended to have more lesions in the right coronary artery and in total than those without.

Abstract S123 Table 1 Comparison of demographic, respiratory disease-related and cardiovascular risk factors between people with and without COPD

\begin{tabular}{|c|c|c|c|}
\hline & No COPD & COPD & $P$ value \\
\hline \multicolumn{4}{|l|}{ Demographics } \\
\hline Number & 203 & 46 & \\
\hline Age (years) & $66 \pm 1$ & $68 \pm 1$ & 0.074 \\
\hline Gender ( $\mathrm{n}(\%)$ male) & $135(67 \%)$ & $37(80 \%)$ & 0.065 \\
\hline Body mass index $\left(\mathrm{kg} / \mathrm{m}^{2}\right)$ & $29.8 \pm 6.9$ & $27.7 \pm 5.2$ & 0.057 \\
\hline \multicolumn{4}{|l|}{ Respiratory disease-related factors } \\
\hline $\mathrm{FEV}_{1} \%$ predicted & $84 \pm 19$ & $68 \pm 2$ & 0.001 \\
\hline Recurrent chest infections ( $\mathrm{n}(\%)$ ) & $19(9 \%)$ & $6(13 \%)$ & 0.304 \\
\hline High sensitivity CRP (mg/L) & $2.1(0.9-5.4)$ & $4.3(1.4-7.5)$ & 0.040 \\
\hline \multicolumn{4}{|l|}{ Traditional cardiovascular risk factors } \\
\hline \multicolumn{4}{|l|}{ Smoking status (pack years) } \\
\hline-10 & $68 \%$ & $0 \%$ & \\
\hline $10-40$ & $26 \%$ & $59 \%$ & \\
\hline$>40$ & $6 \%$ & $41 \%$ & \\
\hline LDL cholesterol (mmol/l) & $2.7+1.0$ & $2.8+1.2$ & 0.429 \\
\hline Systolic blood pressure $(\mathrm{mmHg})$ & $135+19$ & $132+20$ & 0.589 \\
\hline Diabetes (n (\%)) & $67(33 \%)$ & $8(17 \%)$ & 0.025 \\
\hline \multicolumn{4}{|l|}{ Coronary artery disease burden } \\
\hline Gensini score & $12.5(6.0-26.8)$ & $22.5(8.5-46.0)$ & 0.040 \\
\hline Number of vessels affected & $2.2 \pm 1.0$ & $2.4 \pm 1.0$ & 0.125 \\
\hline Total number of lesions & $4.3 \pm 2.6$ & $5.2 \pm 2.7$ & 0.065 \\
\hline - Left coronary artery lesions & $2.0 \pm 1.2$ & $2.1 \pm 1.3$ & 0.385 \\
\hline - Circumflex lesions & $1.0 \pm 0.8$ & $1.3 \pm 1.0$ & 0.028 \\
\hline - Right coronary artery lesions & $1.4 \pm 1.2$ & $1.7 \pm 1.1$ & 0.071 \\
\hline
\end{tabular}

FEV 1 , forced expiratory volume in $1 \mathrm{~s} ; \mathrm{mMRC}$, modified Medical Research Council dyspnoea scale; CAT, COPD assessment test. Values are mean \pm standard deviation, compared with independent $\mathrm{t}$ tests, median (interquartile range), compared with Mann-Whitney $\mathrm{U}$ tests, or number $(\%)$, compared with chi squared tests.

Conclusions People with COPD have more severe coronary artery disease than those without. This analysis cannot determine whether this was due to the presence of COPD or the fact that patients with CAD and COPD had much greater cigarette smoke exposure than CAD patients without COPD.

\section{S124 THE BODE INDEX IS AN INDEPENDENT DETERMINANT OF ARTERIAL STIFFNESS IN CHRONIC OBSTRUCTIVE PULMONARY DISEASE (COPD)}

${ }^{1} \mathrm{M}$ Fisk, ${ }^{2} \mathrm{NS}$ Gale, ${ }^{3} \mathrm{D}$ Mohan, ${ }^{1} \mathrm{CM}$ McEniery, ${ }^{1} \mathrm{JR}$ Forman, ${ }^{4} \mathrm{CE}$ Bolton, ${ }^{5} \mathrm{~W}$ MacNee, ${ }^{2} J R$ Cockcroft, ${ }^{1} \mathrm{~J}$ Fuld, ${ }^{6} \mathrm{PMA}$ Calverley, ${ }^{1} \mathrm{~J}$ Cheriyan, ${ }^{7} \mathrm{R}$ Tal-Singer, ${ }^{3} \mathrm{Ml}$ Polkey, ${ }^{1}$ IB Wilkinson. 'University of Cambridge \& Cambridge University Hospitals NHS Foundation Trust, Cambridge, UK; ${ }^{2}$ Cardiff University, Cardiff, UK; ${ }^{3}$ Royal Brompton \& Harefield NHS Foundation Trust, London, UK; ${ }^{4}$ University of Nottingham, Nottingham, UK; ${ }^{5}$ Edinburgh University, Edinburgh, UK; ${ }^{6}$ University of Liverpool, Liverpool, UK; ${ }^{7}$ GSK, Pennsylvania, USA

\subsection{6/thoraxjnl-2015-207770.130}

Introduction COPD is associated with increased cardiovascular events, independent of established risk factors. Arterial stiffness and carotid intima-media thickness (CIMT) are surrogates of cardiovascular risk and we sought to determine their relationship with COPD severity and prognosis in the ERICA (Evaluation of role of inflammation in airways disease) multi-site UK study: the 
largest cohort study focusing on cardiovascular manifestations in COPD.

Methods Spirometry, haemodynamic measures (aortic pulse wave velocity (aPWV), augmentation index (AIx), peripheral and central blood pressure (BP)) and CIMT (ultrasound measure of carotid artery intima-media layer thickness) were performed in 729 COPD subjects aged $\geq 40$ years. COPD severity was classified by BODE Index [BMI, Obstruction (FEV1), Dyspnoea (mMRC score), Exercise tolerance (6-minute walk distance)], a validated score based on clinical variables and predictor of mortality in COPD.

Results Mean aPWV was 10.3 (SD 2.6) m/s, AIx 27 (10)\%, brachial BP 144/82 (18/11) mmHg, central BP 131/82 (18/11) mmHg, CIMT $0.86(0.4) \mathrm{mm}$.

BODE correlated with aPWV $(\mathrm{p}<0.0001)$ and this was maintained when adjusted for study site, age, supine heart rate (HR) mean arterial pressure (MAP), years smoked and cardiovascular comorbidities (MI, stroke, diabetes, peripheral vascular disease), $\mathrm{p}<0.0001$. BODE was also associated with AIx when adjusted for site, age, seated HR and MAP, years smoked and cardiovascular comorbidities, $\mathrm{p}<0.01$. The constituent variables of BODE did not have the same significant association with both aPWV and AIx, Table 1.

Abstract S124 Table 1 Comparison of linear regression models of BODE constituent variables, cardiovascular comorbidities and established predictors of arterial stiffness

\begin{tabular}{lllll}
\hline Dependent variable & aPWV $(\mathrm{m} / \mathrm{s})$ & & \multicolumn{2}{l}{ Augmentation Index (\%) } \\
\cline { 2 - 5 } & $\boldsymbol{\beta}$ coefficient & $\mathrm{p}$-value & $\boldsymbol{\beta}$ coefficient & $\mathrm{p}$-value \\
\hline Age (years) & 0.4 & $<0.0001$ & -0.01 & 0.7 \\
MAP (mmHg)* & 0.2 & $<0.0001$ & 0.26 & $<0.0001$ \\
HR (bpm)* & 0.2 & $<0.0001$ & -0.49 & $<0.0001$ \\
TPYs & -0.001 & 0.98 & 0.02 & 0.63 \\
BMI (kg/m $\left.{ }^{2}\right)$ & 0.09 & 0.01 & -0.17 & $<0.0001$ \\
FEV1 $(\%)$ & -0.06 & 0.09 & 0.05 & 0.14 \\
mMRC (0-4) & 0.07 & 0.09 & 0.003 & 0.94 \\
6MWD (m) & -0.03 & 0.5 & -0.01 & 0.002 \\
MI & -0.01 & 0.7 & -0.08 & 0.02 \\
Stroke & -0.01 & 0.7 & -0.02 & 0.46 \\
Diabetes & 0.07 & 0.03 & -0.08 & 0.02 \\
PVD & 0.1 & 0.004 & 0.01 & 0.78 \\
Study site & -0.06 & 0.09 & -0.03 & 0.45 \\
\hline
\end{tabular}

*Supine for aortic pulse wave velocity (aPWV), seated for Augmentation Index. TPYs: Total pack years smoked, mMRC dyspnoea scale, 6MWD: 6-minute walk distance, MI: Myocardial Infarction, PVD: Peripheral Vascular Disease.

Cardiovascular comorbidities: self-reported on questionnaire.

An inverse correlation of BODE with central systolic BP ( $\mathrm{p}=$ 0.003 ) was observed and this was maintained after adjustment for study site, age and HR p $=0.03$. There was no significant relationship between BODE and CIMT.

Conclusions BODE is associated with arterial stiffness in COPD, independent of traditional risk factors. Its negative relationship with systolic pressure suggests increasing arterial stiffness with COPD severity, is independent of blood pressure. The BODE Index composite variables are not on the causal pathway for vascular stiffness, so its positive association likely reflects patient susceptibility to injury from smoke or other irritants in the lungs and vasculature. BODE may also enhance cardiovascular risk stratification in COPD, since its relationship with stiffness was independent of self-reported cardiovascular comorbidities.
$\$ 125$

RELATIONSHIP OF RIGHT HEART ECHO PARAMETERS TO FUNCTIONAL STATUS AND PULMONARY FUNCTION IN SEVERE COPD

${ }^{1}$ PSK Liu-Shiu-Cheong, ${ }^{1} B$ L Lipworth, ${ }^{1} A R$ Goudie, ${ }^{1}$ PM Short, ${ }^{2}$ PJ Hopkinson, ${ }^{2}$ AD Struthers. ${ }^{1}$ Scottish Centre for Respiratory Research, Ninewells Hospital, University of Dundee, Dundee, UK: ${ }^{2}$ Division of Cardiovascular and Diabetes Medicine, Ninewells Hospital, University of Dundee, Dundee, UK

\subsection{6/thoraxjnl-2015-207770.131}

Introduction COPD is associated with structural and functional cardiac changes, particularly the right heart. There is little evidence as to whether right heart echo parameters are associated with QOL, health status and pulmonary function.

Methods We looked at the relationship of right heart function to QOL (SGRQ) and health status (SF-36) and to pulmonary function (spirometry and DLCO) in patients with severe COPD.

Results 120 patients were included in the analysis: 82 men and 38 women; mean age 69 years; mean $\mathrm{FEV}_{1} 41 \%$; mean FEV1/ FVC 0.38; mean PAP $29 \mathrm{mmHg}$; mean $\mathrm{SaO}_{2}$ 95\%. Pulmonary vascular resistance was related to $6 \mathrm{MW}$ distance $(\mathrm{p}=0.008)$, BODE Index $(p=0.01)$ as well as FVC\% $(p=0.03)$. RV ejection time (RVET) was related to SGRQ $(p=0.02)$, SF-36 scores for limitations due to physical problems (PL) $(p=0.03)$, social functioning $(\mathrm{SF})(\mathrm{p}=0.04)$ and general health perceptions $(\mathrm{GH})$ $(\mathrm{p}=0.02)$ as well as FVC\% $(\mathrm{p}<0.001)$ and DLCO $(\mathrm{p}=$ 0.001 ). When comparing pulmonary acceleration time (PAT) $<100 \mathrm{~ms}(\mathrm{n}=68)$ vs PAT $\geq 100 \mathrm{~ms}(\mathrm{n}=51)$, we found a difference in mean SGRQ 59.3 vs $52.1(\mathrm{p}=0.01)$ and mean RVET 262 vs $286 \mathrm{~ms}(\mathrm{p}=0.001)$. Dynamic lung volumes as $\mathrm{FEV}_{1} \%, \mathrm{FVC} \%$ and $\mathrm{FEV}_{1} / \mathrm{FVC}$ were significantly related to SGRQ and SF-36 scores for physical function (PF), PL, SF and $\mathrm{GH}$. There were significant differences between GOLD 2 vs GOLD 4 groups for: mean PAP ( $\mathrm{p}=0.05)$, mean RVET ( $\mathrm{p}=$ $0.001)$ and mean SF-36 scores for PF $(\mathrm{p}<0.001)$, PL ( $\mathrm{p}=$ $0.009)$, SF $(p=0.01)$ and GH $(p=0.001)$.

Conclusion In patients with severe COPD, right heart echocardiographic parameters are associated with functional status and dynamic lung volumes.

\section{Basic mechanisms of airways disease}

\section{S126 MEASURING ER PROTEIN MOBILITY DURING ER FRAGMENTATION IN ALPHA-1-ANTITRYPSIN DEFICIENCY}

${ }^{1} \mathrm{JA}$ Dickens, ${ }^{1} \mathrm{~A}$ Ordonez, ${ }^{1} \mathrm{JE}$ Chambers, ${ }^{2} \mathrm{DA}$ Lomas, ${ }^{1} \mathrm{SJ}$ Marciniak. ${ }^{1}$ University of Cambridge, Cambridge, UK; ${ }^{2}$ University College, London, London, UK

\subsection{6/thoraxjnl-2015-207770.132}

Introduction and objectives Alpha-1-antitrypsin is a serine protease inhibitor produced in the liver that is responsible for the regulation of pulmonary inflammation. The commonest pathogenic gene mutation yields Z-alpha-1-antitrypsin, which has a propensity to self-associate into polymers that become entrapped within inclusions of endoplasmic reticulum (ER). This predisposes to the development of cirrhosis, while the resulting paucity of circulating alpha-1-antitrypsin leads to early-onset emphysema. It is unclear whether intracellular inclusions are physically or functionally connected to the main ER network in Z-alpha-1antitrypsin expressing cells. In this study, we sought to clarify the behaviour of proteins within inclusion bodies to further our 